\title{
Photonic Nanojets in Coupled Microcavities
}

\author{
Y.P. Rakovich ${ }^{1}$, D. McCloskey ${ }^{1}$, J.F. Donegan ${ }^{1,2}$ \\ 1. School of Physics, Trinity College Dublin,Dublin 2, Ireland \\ 2. Centre for Research on Adaptive Nanostructures and Nanodevices, Trinity College Dublin,Dublin 2, Ireland
}

Dielectric transparent microspheres are three-dimensional spherical microcavities which provide high Q-factors and a small mode volume leading to strong optical feedback within the cavity [1]. In this present work, we studied the optical properties of symmetrically arranged micrcavities (photonic molecules) with regards to resonance modes and directional beam emission by means of photonic nanojets [2]. The unique feature of nanojets is a directional beam with a beam waist smaller than the diffraction limit. In fact, a transverse beamwidth as small as $0.3 \lambda$ has been reported [3]. In contrast to diffraction-limited microlensing, the nano-jet phenomenon is a near-field effect due to the proximity of the focus position and the microsphere surface. Also because of the nano-scale beam waist, the photonic nano-jets can reach a very high intensity. This offers new opportunities for a broad range of applications in biophotonics, plasmonics, optical data storage and ultramicroscopy. One of the motivations for our research is to examine the feasibility of subdiffractional resolution of imaging and sub-wavelength optical signal waveguiding.

Using finite-element computational approach we have found that a plane-wave-illuminated lossless dielectric microsphere generates a photonic nanojet (Fig.1a) propagating for a distance of approximately one micrometer in the attached microsphere (Fig.1b).
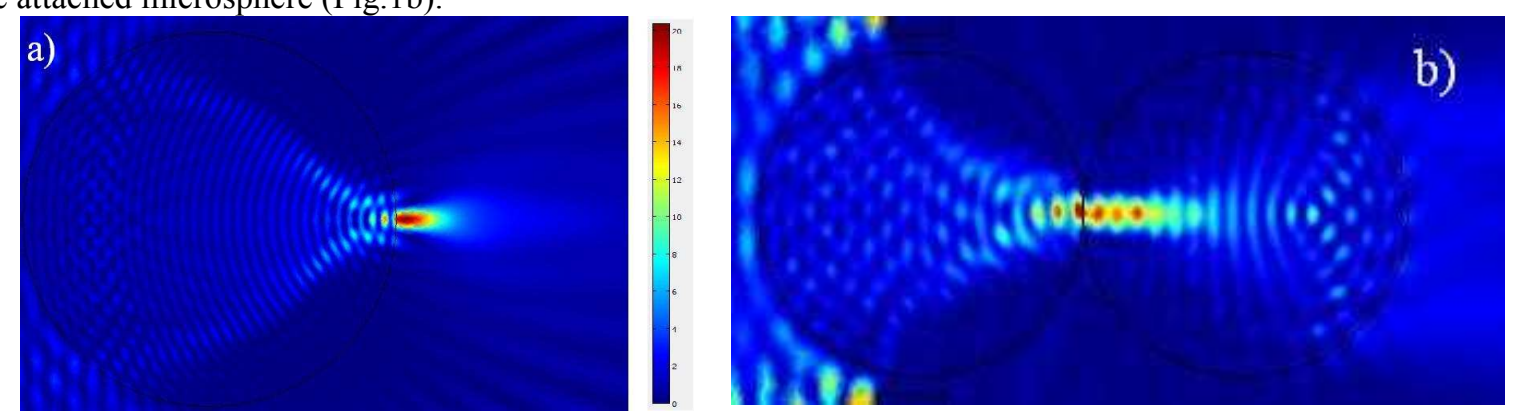

Fig. 1 An example of an individual nanojet (a) and in coupled microcavity structure (b).

Also we experimentally observed strongly directed far field emission in 3-sphere, 5-sphere and 7-sphere structures, which we attribute to photonic nanojets. The directional emission from the photonic molecules matches the symmetry of the structure (Fig. 2) with 3 jets located at 120 degrees with respect to each other for the triangular photonic molecule.

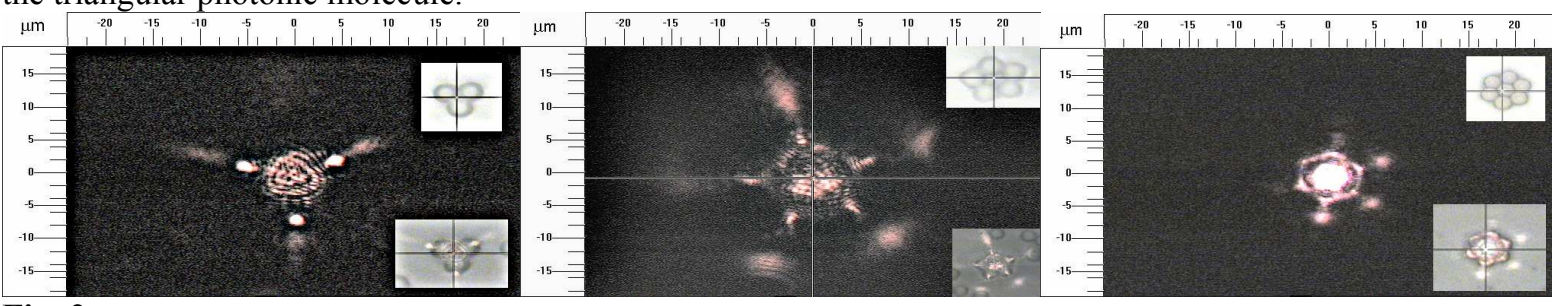

Fig. 2 Images of 3-sphere, 5-sphere and 7-sphere cyclic photonic molecule in white light. The diameter of individual sphere is $5.374 \mu \mathrm{m}$ The structure was illuminated in the centre with a defocused beam $8 \mu \mathrm{m}$ above the substrate.

The most outstanding feature of this structure is the field intensity within the photonic molecules. The detected light from 7-sphere structures forms a hexagonal shape with a strong resemblance to the electron cloud distribution in a chemical benzene molecule.

The observed directed emission suggests the possible use of these photonic structures as a beam splitter with a number of ports corresponding to the number of spheres arranged in the photonic molecule. Photonic nanojets may allow coupling of light from the photonic molecule into other photonic micro- or nanostructure such as coupled resonant optical waveguides or nanostructures such as nanocoaxial cables, which are sub-wavelength optical waveguides. This would be possible not only with sub-wavelength spatial accuracy, but also with extremely high intensities in comparison with conventional sub-wavelength coupling techniques using scanningnear-field optical microscopy-tips.

\section{References}

[1] K.J. Vahala, "Optical microcavities", Nature 424,839 (2003)

[2] S. Lecler, Y. Takakura, P. Meyrueis, "Properties of a three-dimensional photonic jet" Opt. Lett. 302641 (2005).

[3] A. Heifetz, J. J. Simpson, S.-C. Kong, A. Taflove, and V. Backman, "Subdiffraction optical resolution of a

gold nanosphere located within the nanojet of a Mie-resonant dielectric microsphere," Opt. Express 15, 17334 (2007). 